\title{
Effects of High Sugar Content on Fermentation Dynamics and Some Metabolites of Wine-Related Yeast Species Saccharomyces cerevisiae, S. uvarum and Starmerella bacillaris
}

\author{
Borbála Oláhné Horváth*®, \\ Diána Nyitrainé Sárdy®, \\ Nikolett Kellner॰ and \\ Ildikó Magyarø
}

Szent István University Faculty of Horticultural Science Department of Oenology, Ménesi út 45, 1118 Budapest, Hungary

Received: 25 July 2019

Accepted: 18 February 2020

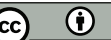

*Corresponding author:

Phone: +3613057347

Fax: +361305700

E-mail: olahne.horvath.borbala@kertk.szie.hu

\section{SUMMARY}

Starmerella bacillaris (synonym Candida zemplinina) is an important non-Saccharomyces yeast in winemaking with valuable oenological properties, accompanying Saccharomyces species in sweet wine fermentation, and has also been suggested for application as combined starter culture in dry or sweet wines. In this study, the major metabolites and nitrogen utilization of these yeasts are evaluated in the musts with high or extremely high sugar concentration. The change in the metabolic footprint of Saccharomyces cerevisiae, Saccharomyces uvarum and Starmerella bacillaris strains was compared when they were present as pure cultures in chemically defined grape juice medium with 220 and $320 \mathrm{~g} / \mathrm{L} \mathrm{of}$ sugar, to represent a fully matured and an overripe grape. Surprisingly, the extreme sugar concentration did not result in a considerable change in the rate of sugar consumption; only a shift of the sugar consumption curves could be noticed for all species, especially for Starmerella bacillaris. At the extreme sugar concentration, Starmerella bacillaris showed excellent glycerol production, moderate nitrogen demand together with a noticeable proline utilisation. The change in the overall metabolite pattern of Starmerella bacillaris allowed clear discrimination from the change of the Saccharomyces species. In this experiment, the adequacy of this non-Saccharomyces yeast for co-fermentation in juices with high sugar concentration is highlighted. Moreover, the results suggest that Starmerella bacillaris has a more active adaptation mechanism to extremely high sugar concentration.

Key words: Starmerella bacillaris, Candida zemplinina, non-Saccharomyces yeast, high sugar concentration, metabolic footprint

\section{INTRODUCTION}

Fermentation of natural sweet wines has always been a big challenge in winemaking, but the global changes in the climate influence the parameters of the raw grape juice, and as a consequence the quality of the dry or semi-sweet wines. The higher initial sugar concentration is one of the most important factors involved, which could be a considerable challenge for the yeasts. Moreover, not only the fermentation dynamics and ethanol concentration but also the whole metabolite-profile could be modified remarkably $(1,2)$. In making some special sweet wines like botrytized or straw wines, sugar content of the grapes may reach an extremely high level, multiplying these effects.

Using starter cultures for wine fermentation originally meant exclusively single-strain (Saccharomyces cerevisiae or occasionally Saccharomyces uvarum) products. During the last decade, the industrial exploitation of selected non-Saccharomyces yeasts in combination with Saccharomyces strains as oligo-starter cultures has become an emerging trend (35). One of the promising candidates is Starmerella bacillaris (syn. Candida zemplinina) (2).

S. bacillaris was originally described in Tokaj wine region in Hungary (6) as Candida zemplinina and renamed by Duarte et al. (7). This species is particularly associated with sweet, botrytized wine fermentations $(8,9)$, where it spontaneously co-exists with $S$. cerevisiae and S. uvarum, but it is frequently isolated from other wine-related sources $(10,11)$. From oenological aspect, this species has several valuable properties like high glycerol production, fructophilic character and outstanding osmotolerance, reviewed by Englezos et al. (12). 
The major metabolite pattern, in terms of particular fermentation by-products, plays a significant role in forming the final wine quality, while a moderate nitrogen demand, osmotolerance and fructophilic behaviour could help to avoid sluggish or stuck fermentation. These properties are highly varying on interspecific level, although there could be a considerable difference on intraspecific level too. The conventional wine yeasts, S. cerevisiae and S. uvarum are well-characterized from this aspect $(13,14)$, but $S$. bacillaris along with other non-conventional yeasts have only recently been in the focus of wine research (e.g. 15,16). Going one step further, a relevant question is how an extreme sugar concentration can modify the above-described character of different wine yeasts.

The aim of this study is to compare and contrast the changes in some major metabolite products and nitrogen utilization induced by extremely high $(320 \mathrm{~g} / \mathrm{L})$ initial sugar concentration with a normal, but still considerable $(220 \mathrm{~g} / \mathrm{L})$, sugar level of the two most important wine yeasts Saccharomyces cerevisiae and Saccharomyces uvarum, and Starmerella bacillaris. Although this last species has been thoroughly studied in recent years at moderate sugar concentrations, to our knowledge, only a few studies investigated high sugar concentrations (17-19). In our work, we performed a direct comparison among the three species. The metabolites and substrates analysed in this paper were chosen on the basis of their outstanding oenological importance and included the ethanol, glycerol, volatile acidity, L-malic acid, L-succinic acid, yeast assimilable nitrogen and proline. The emphasis was on the effects of extremely high sugar concentration on these non-volatile compounds, but the behaviour of the different yeast species was also compared.

\section{MATERIALS AND METHODS}

\section{Yeast strains}

Strains of three yeast species used in this study are as follows: three Starmerella bacillaris strains: Y1667 (CBS9494 type strain) and Y1756 from the National Collection of Agricultural and Industrial Microorganisms (NCAIM), Budapest, Hungary, isolated from botrytized Tokaj grape, and strain MLO from the Department of Oenology, Szent István University (DOSZIU) culture collection, Budapest, Hungary, isolated from wine, six Saccharomyces cerevisiae strains: UVAFERMPM and UVAFERM228 commercial wine yeasts, strains SC57, RA100 and SB12 from DO-SZIU, isolated from Tokaj Aszú wine, and strain S701 also from DO-SZIU, isolated from the wine from Somló wine region, and three Saccharomyces uvarum strains: CBS395, type strain from NCAIM, SB42 from DO-SZIU, both isolated from Tokaj Aszú wine, and S103 from DO-SZIU, isolated from the wine from Somló wine region. The natural isolates were previously identified at the Department of Microbiology and Biotechnology, Szent István University (20).

\section{Culture media}

The fermentation was carried out in chemically defined grape juice medium at 220 and $320 \mathrm{~g} / \mathrm{L}$ of sugar. Based on
Henschke and Jiranek (21), the medium included (per litre of distilled water) D-glucose 110 or $160 \mathrm{~g}$, D-fructose 110 or $160 \mathrm{~g}$, $\mathrm{KOH} 4 \mathrm{~mL}$ to adjust $\mathrm{pH}$ to 3.3, yeast carbon base $11.7 \mathrm{~g}$, Tween $800.5 \mathrm{~mL}$, L-tartaric acid $2 \mathrm{~g}$ and L-malic acid $3 \mathrm{~g}$. To obtain the yeast assimilable nitrogen (YAN) level of $300 \mathrm{mg} / \mathrm{L}$, the following mixture of single amino acids and diammonium phosphate was used (in $\mathrm{mg} / \mathrm{L}$ in the final medium): arginine 657.0, asparagine 131.0, aspartic acid 263.0, glutamine 175.0, glutamic acid 438.0, histidine 8.8, isoleucine 20.5, leucine 67.0, methionine 44.0, phenylalanine 17.5 , threonine 43.9, tryptophan 17.5, tyrosine 17.5, valine 87.7 and proline 438.5, which is not part of YAN but is present in every grape juice, and diammonium phosphate 136.0. All chemicals were obtained from Sigma-Aldrich Chemie GmbH, Merck, Munich, Germany.

\section{Yeast culture maintenance}

Yeast cultures were maintained on YEPD ( $20 \mathrm{~g} / \mathrm{L}$ glucose, $10 \mathrm{~g} / \mathrm{L}$ peptone, $10 \mathrm{~g} / \mathrm{L}$ yeast extract and $15 \mathrm{~g} / \mathrm{L}$ agar-agar) agar slants and stored at $6{ }^{\circ} \mathrm{C}$. For inoculum preparation, an inoculation loop of cells from fresh agar slants was transferred into $20 \mathrm{~mL}$ of YEPD broth in 100-mL flasks and incubated at $25^{\circ} \mathrm{C}$ for $48 \mathrm{~h}$ without agitation.

\section{Fermentation conditions}

Fermentations were carried out under semi-anaerobic conditions, in 200-mL flasks containing $180-\mathrm{mL}$ aliquots of culture media, without shaking, in triplicate. The flasks were inoculated with $10^{6} \mathrm{cell} / \mathrm{mL}$ with $3 \%$ of 48 -hour-old yeast cultures grown in YEPD broth. The fermentation temperature was kept at $20^{\circ} \mathrm{C}$. The course of fermentation was monitored by measuring the total soluble solids content (Brix values) with an Atago RX-5000 CX digital refractometer (Atago Co. Ltd., Fukaya-shi, Japan) after filtration, by sampling on days 0,2 , $4,7,9,14,21$ and 28. Biomass concentration was measured by Bürker chamber cell counting after methylene blue staining. After the monitoring period of 28 days, the samples for chemical analysis were membrane filtered $(0.45 \mu \mathrm{m})$ and stored at $-18^{\circ} \mathrm{C}$ until analysis.

\section{Chemical and statistical analysis}

Ethanol concentrations were measured by distillation and determination of specific gravity of the distillate (22), volatile acidity (expressed as acetic acid) by steam distillation followed by titration (23) and reducing sugars by iodometric titration (24) using the official methods of the International Organisation of Vine and Wine. D-glucose/D-fructose ratio (K-FRUGL), glycerol (K-GCROL), L-succinic acid (K-SUCC), L-malic acid (K-LMAL) and YAN (K-LARGE and K-PANOPA) concentrations were measured with Megazyme enzymatic kits (Bray, Ireland). Proline concentrations were measured by ${ }^{1} \mathrm{H}$ NMR technique on a Bruker AVANCE 400 spectrometer and 400'54 ASCEND magnet system (Bruker, Karlsruhe, Germany) (25). 
Data were evaluated with analysis of variance one way (ANOVA) and multivariate (MANOVA) and discriminant analysis (DA), after checking the assumptions, using the statistical package IBM SPSS Statistics for Windows v. 23.0 (26).

\section{RESULTS AND DISCUSSION}

\section{Sugar consumption dynamics}

Sugar consumption curves of the different species are shown in Fig. 1. At the end of the monitored period of fermentation with the lower initial sugar concentration (220 $\mathrm{g} / \mathrm{L}$ ), only S. cerevisiae strains UVAFERMPM, SC57 and S701 completed the fermentation. After fermentation with S. cerevisiae strains RA100, UVAFERM 228 and SB12, smaller amounts of sugar remained (13.6-22.2 g/L), while with all the S. uvarum and S. bacillaris strains, considerable amounts of sugar remained (45.1-75.3 and 117.5-125.0 g/L respectively), which is in accordance with the earlier described behaviour of the given species (9).

The most striking observation is that the extreme sugar concentration $(320 \mathrm{~g} / \mathrm{L})$ did not influence significantly the rate of sugar consumption by S. uvarum, and in particular by S. bacillaris strains. Only a shift can be noticed in the sugar consumption curves, according to the elevated initial sugar content of the juice, resulting in higher residual sugar concentrations (Fig. 1).

\section{Effect of extreme sugar concentration on the major metabolites}

Our focus was on the major by-products of the alcoholic fermentation present in the chemically defined grape juice at the end of the monitored period (28 days) (Table 1). This experiment confirmed the earlier described strong fructophilic nature of S. bacillaris $(8,9)$ (Table 1), and this characteristic was not influenced by the high sugar concentration, therefore, it was excluded from further analysis and the sum of the consumed glucose and fructose was used for the yields, if applicable (Table 2 and Table 3).

Starmerella bacillaris strains used the highest concentration of sugars to produce $1 \%$ ethanol, the Saccharomyces strains used considerably less (Table 2). Evaluating the change, only S. cerevisiae S701 was able to produce slightly increased alcohol yield from the excess sugar. The sugar-induced change in the ethanol yield was only significant when using S. bacillaris Y1667 and MLO (reduction from 0.34 to 0.27 $0.30 \mathrm{~g} / \mathrm{g}$ ), which implies that these strains changed their main metabolite ratio due to the higher osmotic pressure.

The glycerol yield of S. bacillaris strains was considerably higher than of the investigated Saccharomyces species at both sugar levels (Table 2). The strong positive effect of the increasing sugar concentration was confirmed previously for the S. cerevisiae and S. uvarum strains and was verified also for S. bacillaris in this study, in accordance with the findings of Rantsiou et al. (18). The high glycerol production of the S. bacillaris strains
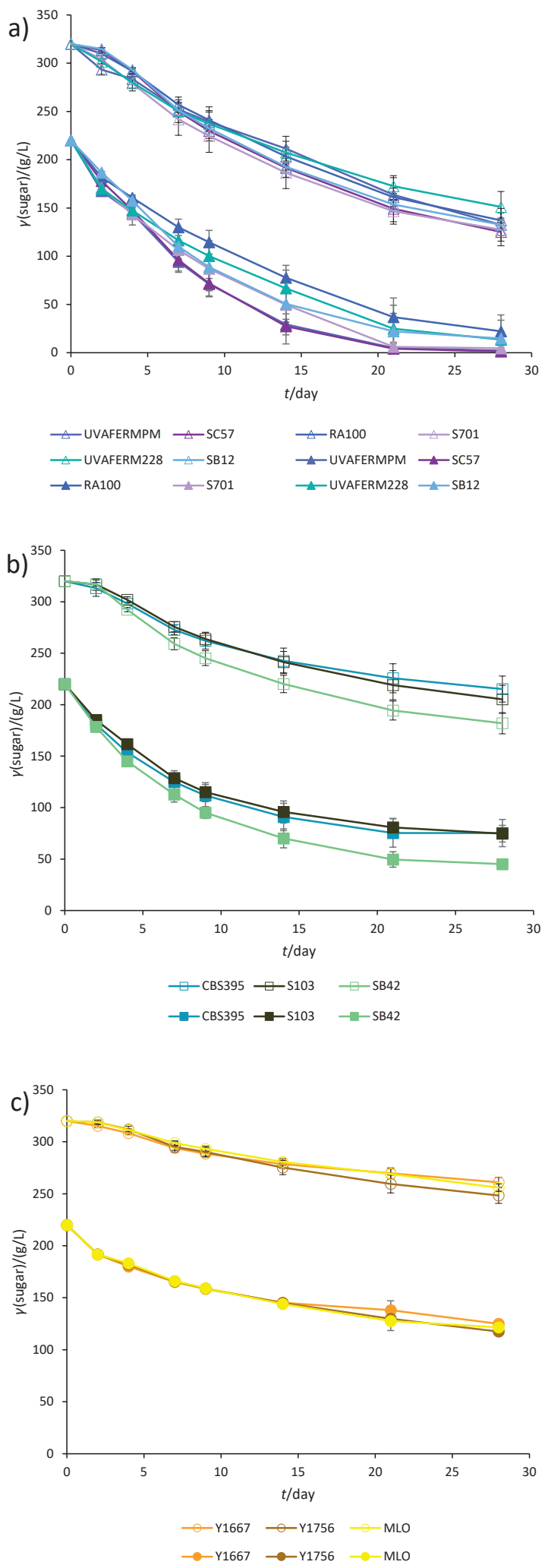

Fig. 1. Sugar consumption dynamics of the investigated strains in chemically defined grape juice: a) Saccharomyces cerevisiae, b) S. uvarum, and c) Starmerella bacillaris. Full symbols: initial $\gamma$ (sugar) $=220 \mathrm{~g} / \mathrm{L}$, empty symbols: initial $\gamma$ (sugar) $=320 \mathrm{~g} / \mathrm{L}$. Data are mean values of the triplicate fermentations with standard deviations $(N=3)$ 
Table 1. Major metabolites and cell density of chemically defined grape juice fermented with different yeast species at initial $\gamma($ sugar) $=220$ and $320 \mathrm{~g} / \mathrm{L}$

\begin{tabular}{|c|c|c|c|c|c|c|c|c|c|}
\hline $\begin{array}{c}\gamma(\text { sugar }) / \\
\text { (g/L) }\end{array}$ & Species & Strain & $\varphi($ ethanol $) / \%$ & $\begin{array}{c}\gamma(\text { glycerol }) / \\
(\mathrm{g} / \mathrm{L})\end{array}$ & $\begin{array}{c}\gamma(\text { acetic acid }) / \\
(\mathrm{g} / \mathrm{L})\end{array}$ & $\begin{array}{c}\gamma(\text { consumed } \\
\text { malic acid }) /(g / L)\end{array}$ & $\begin{array}{l}\gamma(\text { succinic } \\
\text { acid }) /(g / L)\end{array}$ & $\begin{array}{c}\text { Glucose- } \\
\text { fructose ratio }\end{array}$ & $\mathrm{N} \cdot 10^{8} /($ cell $/ \mathrm{mL}$ ) \\
\hline \multirow{17}{*}{220} & \multirow{7}{*}{ 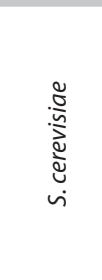 } & UVAFERMPM & $(12.9 \pm 0.2)^{d}$ & $(4.7 \pm 0.3)^{\mathrm{a} *}$ & $(0.72 \pm 0.01)^{c *}$ & $(0.7 \pm 0.1)^{\mathrm{abcd}}$ & $(0.44 \pm 0.01)^{c *}$ & $(0.3 \pm 0.2)^{a}$ & $(2.7 \pm 1.2)^{\mathrm{ab}}$ \\
\hline & & SC57 & $(12.8 \pm 0.2)^{\mathrm{d}}$ & $(4.7 \pm 0.5)^{\mathrm{a} *}$ & $(0.68 \pm 0.07)^{\mathrm{bcd} *}$ & $\left(0.6 \pm 0.2^{\mathrm{ab}}\right.$ & $(0.32 \pm 0.01)^{\mathrm{b}}$ & $(0.8 \pm 0.4)^{\mathrm{a}}$ & $(2.7 \pm 0.3)^{\mathrm{a}}$ \\
\hline & & S701 & $(12.6 \pm 0.2)^{\mathrm{cd} *}$ & $(5.1 \pm 0.3)^{a b *}$ & $(0.78 \pm 0.02)^{c *}$ & $(0.6 \pm 0.1)^{\mathrm{abc}}$ & $(0.31 \pm 0.02)^{\mathrm{ab}}$ & $(0.08 \pm 0.01)^{\mathrm{a}}$ & $(2.0 \pm 0.6)^{\mathrm{ab}}$ \\
\hline & & RA100 & $(11.2 \pm 2.0)^{\mathrm{cd}}$ & $(5.0 \pm 0.3)^{\mathrm{ab} *}$ & $(0.76 \pm 0.05)^{\mathrm{cd} *}$ & $(0.3 \pm 0.1)^{\mathrm{a}}$ & $(0.25 \pm 0.05)^{b}$ & $(0.4 \pm 0.2)^{\mathrm{a}}$ & $(1.19 \pm 0.07)^{\mathrm{ab}}$ \\
\hline & & UVAFERM 228 & $(11.6 \pm 1.3)^{\mathrm{cd}}$ & $(4.9 \pm 0.8)^{\mathrm{a} *}$ & $(0.70 \pm 0.06)^{\mathrm{cd} *}$ & $(0.49 \pm 0.06)^{\mathrm{ab}}$ & $(0.29 \pm 0.04)^{\mathrm{ab}}$ & $(0.18 \pm 0.01)^{\mathrm{a}}$ & $(2.2 \pm 0.3)^{\mathrm{ab}}$ \\
\hline & & SB12 & $(13.1 \pm 0.2)^{\mathrm{cd}}$ & $(5.45 \pm 0.07)^{\mathrm{ab} *}$ & $(1.00 \pm 0.00)^{\mathrm{d} *}$ & $(0.46 \pm 0.00)^{\mathrm{a}}$ & $(0.31 \pm 0.02)^{\mathrm{a}}$ & $(0.5 \pm 0.3)^{\mathrm{a}}$ & $(1.4 \pm 0.5)^{\mathrm{ab}}$ \\
\hline & & Mean & $(12.4 \pm 1.4)$ & $(5.0 \pm 0.5)$ & $(0.8 \pm 0.1)$ & $(0.5 \pm 0.2)$ & $(0.32 \pm 0.07)$ & $(0.4 \pm 0.3)$ & $(2.0 \pm 0.7)$ \\
\hline & \multirow{4}{*}{$\begin{array}{l}\xi \\
\frac{\xi}{2} \\
\vdots \\
3 \\
\text { vi }\end{array}$} & CBS395 & $(7.6 \pm 0.7)^{\mathrm{bc}}$ & $(5.8 \pm 0.4)^{a b *}$ & $(0.52 \pm 0.03)^{b *}$ & $(0.73 \pm 0.06)^{\mathrm{bcd} *}$ & $(0.31 \pm 0.03)^{\mathrm{b} *}$ & $(0.39 \pm 0.07)^{\mathrm{a}}$ & $(1.2 \pm 0.2)^{b}$ \\
\hline & & $\mathrm{S} 103$ & $(7.6 \pm 0.4)^{\mathrm{bc}}$ & $(6.5 \pm 0.8)^{\mathrm{abc} *}$ & $(0.46 \pm 0.03)^{\mathrm{b} *}$ & $(0.59 \pm 0.06)^{\mathrm{abc}}$ & $(0.26 \pm 0.05)^{\mathrm{ab}}$ & $(0.39 \pm 0.04)^{\mathrm{a}}$ & $(1.74 \pm 0.09)^{\mathrm{ab}}$ \\
\hline & & SB42 & $(9.9 \pm 0.5)^{\mathrm{bc}}$ & $(5.4 \pm 0.4)^{\mathrm{ab} *}$ & $(0.28 \pm 0.01)^{a *}$ & $(0.73 \pm 0.06)^{\mathrm{bcd} *}$ & $(0.27 \pm 0.04)^{\mathrm{ab}}$ & $(0.20 \pm 0.02)^{\mathrm{a}}$ & $(1.7 \pm 0.2)^{\mathrm{ab}}$ \\
\hline & & Mean & $(8.4 \pm 1.2)$ & $(5.9 \pm 0.7)$ & $(0.4 \pm 0.1)$ & $(0.68 \pm 0.09)$ & $(0.28 \pm 0.04)$ & $(0.3 \pm 0.1)$ & $(1.5 \pm 0.3)$ \\
\hline & \multirow{4}{*}{ 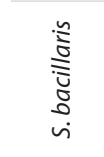 } & Y1667 & $(4.4 \pm 0.2)^{\mathrm{a}}$ & $(7.8 \pm 0.7)^{b c *}$ & $(0.8 \pm 0.1)^{\mathrm{cd}}$ & $(0.9 \pm 0.1)^{\text {cde }}$ & $(0.35 \pm 0.02)^{\mathrm{ab} *}$ & $(4.9 \pm 1.1)^{\mathrm{b}}$ & $(2.1 \pm 0.5)^{\mathrm{ab}}$ \\
\hline & & Y1756 & $(4.8 \pm 0.3)^{\mathrm{a}}$ & $(7.8 \pm 0.2)^{b c *}$ & $(0.74 \pm 0.08)^{c *}$ & $(1.09 \pm 0.06)^{\mathrm{e*}}$ & $(0.40 \pm 0.03)^{\mathrm{ab}}$ & $(5.8 \pm 1.4)^{\mathrm{b}}$ & $(2.0 \pm 05)^{\mathrm{ab}}$ \\
\hline & & MLO & $(4.6 \pm 0.3)^{\mathrm{a}}$ & $(7.4 \pm 0.6)^{c *}$ & $(0.7 \pm 0.1)^{\mathrm{bcd} *}$ & $(0.96 \pm 0.1)^{\mathrm{de} *}$ & $(0.40 \pm 0.03)^{\mathrm{ab} *}$ & $(6.0 \pm 1.7)^{b}$ & $(2.0 \pm 0.2)^{\mathrm{ab}}$ \\
\hline & & Mean & $(4.6 \pm 0.3)$ & $(7.6 \pm 0.5)$ & $(0.7 \pm 0.1)$ & $(1.0 \pm 0.1)$ & $(0.39 \pm 0.03)$ & $(5.6 \pm 1.3)$ & $(2.0 \pm 0.4)$ \\
\hline & \multicolumn{2}{|c|}{$\mathrm{LSD}_{5 \% \mathrm{~S} \text {.c-other }}$} & 1.0 & 0.45 & 0.09 & 0.11 & n.s. & 0.57 & n.s. \\
\hline & \multicolumn{2}{|c|}{$\mathrm{LSD}_{5 \% \text { S. u-s.b. }}$} & 1.1 & 0.52 & 0.11 & 0.13 & n.s. & 0.65 & n.s. \\
\hline \multirow{17}{*}{320} & \multirow{7}{*}{ 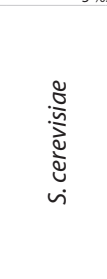 } & UVAFERMPM & $(11.3 \pm 1.3)^{\mathrm{BCD}}$ & $(9.0 \pm 0.3)^{A *}$ & $(1.23 \pm 0.06)^{c *}$ & $(0.53 \pm 0.06)^{A}$ & $(0.35 \pm 0.05)^{\mathrm{ABC} *}$ & $(0.46 \pm 0.05)^{A}$ & $(1.2 \pm 0.2)^{\mathrm{AB}}$ \\
\hline & & SC57 & $(14.3 \pm 1.2)^{\mathrm{D}}$ & $(8.7 \pm 0.2)^{A *}$ & $(1.30 \pm 0.00)^{\mathrm{C} *}$ & $(0.4 \pm 0.1)^{\mathrm{A}}$ & $(0.30 \pm 0.02)^{\mathrm{A}}$ & $(0.47 \pm 0.03)^{\mathrm{A}}$ & $(1.8 \pm 0.6)^{\mathrm{AB}}$ \\
\hline & & $\mathrm{S} 701$ & $(13.6 \pm 0.4)^{\mathrm{D} *}$ & $(9.6 \pm 0.4)^{A *}$ & $(1.63 \pm 0.06)^{\mathrm{D*}}$ & $(0.49 \pm 0.06)^{\mathrm{A}}$ & $(0.30 \pm 0.02)^{\mathrm{A}}$ & $(0.34 \pm 0.03)^{A}$ & $(1.7 \pm 0.4)^{\mathrm{A}}$ \\
\hline & & RA100 & $(13.2 \pm 0.6)^{\mathrm{D}}$ & $(8.4 \pm 0.4)^{A *}$ & $(1.3 \pm 0.1)^{\mathrm{BCD} *}$ & $(0.3 \pm 0.1)^{\mathrm{A}}$ & $(0.22 \pm 0.00)^{\mathrm{AB}}$ & $(0.61 \pm 0.03)^{A}$ & $(1.0 \pm 0.2)^{\mathrm{AB}}$ \\
\hline & & UVAFERM 228 & $(11.5 \pm 2.8)^{A B C D}$ & $(8.2 \pm 0.6)^{A *}$ & $(1.3 \pm 0.2)^{B C D *}$ & $(0.6 \pm 0.2)^{A}$ & $(0.27 \pm 0.05)^{A B}$ & $(0.44 \pm 0.09)^{A}$ & $(2.4 \pm 0.3)^{A B}$ \\
\hline & & SB12 & $(13.3 \pm 0.3)^{\mathrm{CD}}$ & $(10.6 \pm 0.2)^{\mathrm{CDE} *}$ & $(1.8 \pm 0.0)^{\mathrm{D*}}$ & $(0.43 \pm 0.06)^{\mathrm{A}}$ & $(0.27 \pm 0.02)^{A B}$ & $(0.41 \pm 0.02)^{\mathrm{A}}$ & $(1.3 \pm 0.5)^{A B}$ \\
\hline & & Mean & $(12.9 \pm 1.6)$ & $(9.1 \pm 0.9)$ & $(1.4 \pm 0.2)$ & $(0.5 \pm 0.1)$ & $(0.28 \pm 0.05)$ & $(0.46 \pm 0.09)$ & $(1.6 \pm 0.6)$ \\
\hline & \multirow{4}{*}{$\begin{array}{l}\frac{5}{3} \\
\frac{5}{5} \\
3 \\
\dot{v}\end{array}$} & CBS395 & $(6.5 \pm 1.2)^{\mathrm{AB}}$ & $(8.3 \pm 0.4)^{A B *}$ & $(0.86 \pm 0.00)^{\mathrm{ABC} *}$ & $(0.49 \pm 0.06)^{A *}$ & $(0.21 \pm 0.02)^{B C *}$ & $(0.69 \pm 0.05)^{A}$ & $(0.9 \pm 0.2)^{\mathrm{A}}$ \\
\hline & & $\mathrm{S} 103$ & $(8.1 \pm 1.3)^{\mathrm{ABC}}$ & $(8.6 \pm 1.0)^{A *}$ & $(0.74 \pm 0.01)^{\mathrm{A} *}$ & $(0.53 \pm 0.06)^{\mathrm{A}}$ & $(0.21 \pm 0.03)^{\mathrm{AB}}$ & $(0.57 \pm 0.06)^{A}$ & $(1.26 \pm 0.07)^{\mathrm{A}}$ \\
\hline & & SB42 & $(10.2 \pm 0.8)^{\mathrm{BCD}}$ & $(9.0 \pm 0.8)^{\mathrm{AC} *}$ & $(0.67 \pm 0.02)^{A *}$ & $(0.46 \pm 0.00)^{A *}$ & $(0.26 \pm 0.03)^{c}$ & $(0.45 \pm 0.03)^{\mathrm{A}}$ & $(2.0 \pm 0.4)^{A B}$ \\
\hline & & Mean & $(8.3 \pm 1.9)$ & $(8.7 \pm 0.7)$ & $(0.75 \pm 0.08)$ & $(0.49 \pm 0.05)$ & $(0.23 \pm 0.04)$ & $(0.57 \pm 0.1)$ & $(1.4 \pm 0.5)$ \\
\hline & \multirow{4}{*}{ 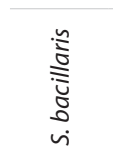 } & Y1667 & $(3.9 \pm 0.3)^{A}$ & $(11.5 \pm 0.8)^{\mathrm{BDE} *}$ & $(1.0 \pm 0.1)^{c}$ & $(0.7 \pm 0.2)^{A}$ & $(0.29 \pm 0.02)^{B C *}$ & $(2.2 \pm 0.2)^{\mathrm{B}}$ & $(2.3 \pm 0.4)^{\mathrm{AB}}$ \\
\hline & & Y1756 & $(4.9 \pm 0.4)^{\mathrm{A}}$ & $(12.0 \pm 1.5)^{\mathrm{BE} *}$ & $(1.2 \pm 0.3)^{\mathrm{BCD} *}$ & $(0.7 \pm 0.2)^{A *}$ & $(0.35 \pm 0.05)^{c}$ & $(2.8 \pm 0.5)^{\mathrm{B}}$ & $(2.1 \pm 0.2)^{\mathrm{B}}$ \\
\hline & & MLO & $(4.2 \pm 0.3)^{\mathrm{A}}$ & $(10.9 \pm 1.0)^{\mathrm{BCDE} *}$ & $(0.85 \pm 0.01)^{\mathrm{ABC} *}$ & $(0.49 \pm 0.06)^{A *}$ & $(0.31 \pm 0.04)^{\mathrm{BC} *}$ & $(2.4 \pm 0.2)^{\mathrm{B}}$ & $(1.9 \pm 0.3)^{\mathrm{AB}}$ \\
\hline & & Mean & $(4.3 \pm 0.5)$ & $(11.5 \pm 1.1)$ & $(1.0 \pm 0.2)$ & $(0.6 \pm 0.2)$ & $(0.32 \pm 0.04)$ & $(2.5 \pm 0.4)$ & $(2.1 \pm 0.3)$ \\
\hline & \multicolumn{2}{|c|}{$\mathrm{LSD}_{5 \% 5 . c-\text { other }}$} & 1.2 & 0.75 & 0.17 & n.s. & n.s. & 0.17 & n.s. \\
\hline & \multicolumn{2}{|c|}{$\mathrm{LSD}_{5 \% \text { s.u.s.b. }}$} & 1.4 & 0.87 & 0.20 & n.s. & n.s. & 0.19 & n.s. \\
\hline
\end{tabular}

Values are mean of triplicate fermentations for each strain, bold values are species mean values of strain performance. According to the Games-Howell post hoc comparison test, the values in the same column are statistically different at $p<0.05$; different lower-case letters indicate difference at $\gamma$ (sugar) $=220 \mathrm{~g} / \mathrm{L}$, capital letters at $\gamma$ (sugar) $=320 \mathrm{~g} / \mathrm{L}$, and asterisks denote the change induced by initial sugar concentration in the grape. $\mathrm{LSD}_{5 \%}$ values are the least significant differences between the mean values of species: $\mathrm{LSD}_{5 \% \text { s.c-other }}$ between Saccharomyces cerevisiae and the other two species, and $\mathrm{LSD}_{5 \% \text { s.u-s.b. }}$ between S. uvarum and Starmerella bacillaris. n.s.=not significant

is highly valuable and it could shape considerably the body of the wine regardless of the sugar level.

The glycerol/ethanol ratio also showed an alteration in the main balance of the alcoholic fermentation, which was 2 to 4 times higher when using S. bacillaris than Saccharomyces species and clearly distinct at both sugar levels. Consequently, reduced alcohol content and a fuller wine texture could be reached employing $S$. bacillaris strains, preferably in combination with Saccharomyces yeasts due to their limited fermentation ability.

It has long been known that the increasing osmotic stress increases the acetic acid production of Saccharomyces species and we found similar trend for S. bacillaris. S. uvarum strains SB42 and S103 had the smallest increase, while CBS395 was similar to S. bacillaris strains Y1667, Y1756 and MLO, followed by S. cerevisiae strains UVAFERMPM, SC57, RA100 and UVAFERM228 (Table 1). In contrast to these results, Rantsiou et al. (18) found a sugar-independent trend in the acetic acid production by S. bacillaris that was mainly time-dependent. The absolute amount of acetic acid is comparable with that produced by Saccharomyces species, which is in accordance with some earlier results (9). Considering the limited fermentation ability of $S$. bacillaris, the acetic acid yield is not problematic since this species is not used in pure culture but in mixed fermentation $(15,16)$.

All species consumed L-malic acid and produced L-succinic acid, at both sugar concentrations. The effect of sugar concentration on the consumption was significant in the case of S. uvarum CBS395 and SB42, and S. bacillaris Y1756 and MLO, resulting in a reduction of the consumed malic acid. The negative initial sugar dependence of L-malic acid utilisation is in 
Table 2. Yields of the main fermentation products calculated from the major metabolites of the different yeast species in chemically defined grape juice at initial $\gamma($ sugar $)=220$ and $320 \mathrm{~g} / \mathrm{L}$

\begin{tabular}{|c|c|c|c|c|c|c|c|c|}
\hline $\begin{array}{c}\gamma \text { (sugar)/ } \\
(\mathrm{g} / \mathrm{L})\end{array}$ & Species & Strain & $Y($ ethanol $) /(\mathrm{g} / \mathrm{g})$ & $m$ (sugar)/g & $Y($ glycerol)/(g/g) & $\begin{array}{c}Y(\text { glycerol/ } \\
\text { ethanol }) /(\mathrm{g} / \mathrm{g})\end{array}$ & $\begin{array}{c}Y(\text { acetic acid }) / \\
(\mathrm{mg} / \mathrm{g})\end{array}$ & $\begin{array}{c}Y(\text { succinic acid }) / \\
(\mathrm{g} / \mathrm{g})\end{array}$ \\
\hline \multirow{17}{*}{220} & \multirow{7}{*}{ 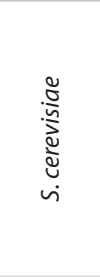 } & UVAFERMPM & $(0.47 \pm 0.01)^{c}$ & $(16.9 \pm 0.2)^{\mathrm{a}}$ & $(0.02 \pm 0.00)^{\mathrm{a} *}$ & $(0.05 \pm 0.00)^{\mathrm{a} *}$ & $(3.29 \pm 0.04)^{b}$ & $(2.01 \pm 0.02)^{\mathrm{b} *}$ \\
\hline & & SC57 & $(0.46 \pm 0.01)^{c}$ & $(17.1 \pm 0.3)^{a}$ & $(0.02 \pm 0.00)^{\mathrm{a} *}$ & $(0.05 \pm 0.00)^{a *}$ & $(3.1 \pm 0.3)^{\mathrm{bc}}$ & $(1.47 \pm 0.04)^{\mathrm{a}}$ \\
\hline & & S701 & $(0.46 \pm 0.01)^{\mathrm{bc}}$ & $(17.1 \pm 0.3)^{\mathrm{a}}$ & $(0.02 \pm 0.00)^{a *}$ & $(0.05 \pm 0.01)^{\mathrm{a*}}$ & $(3.7 \pm 0.1)^{b c *}$ & $(1.6 \pm 0.1)^{\mathrm{ab}}$ \\
\hline & & RA100 & $(0.45 \pm 0.07)^{c}$ & $(17.7 \pm 3.0)^{a}$ & $(0.03 \pm 0.00)^{\mathrm{ab} *}$ & $(0.06 \pm 0.01)^{\mathrm{ab} *}$ & $(3,9 \pm 0.3)^{b}$ & $(1.4 \pm 0.2)^{\mathrm{ab}}$ \\
\hline & & UVAFERM228 & $(0.44 \pm 0.04)^{b c}$ & $(18.2 \pm 1.9)^{\mathrm{a}}$ & $(0.02 \pm 0.00)^{a *}$ & $(0.05 \pm 0.00)^{\mathrm{ab} *}$ & $(3.4 \pm 0.3)^{\mathrm{b} *}$ & $(1.4 \pm 0.2)^{\mathrm{a}}$ \\
\hline & & SB12 & $(0.47 \pm 0.01)^{c}$ & $(16.8 \pm 0.2)^{\mathrm{a}}$ & $(0.03 \pm 0.00)^{\mathrm{a} *}$ & $(0.05 \pm 0.00)^{\mathrm{ab} *}$ & $(4.58 \pm 0.01)^{c *}$ & $(1.4 \pm 0.1)^{\mathrm{a} *}$ \\
\hline & & Mean & $(0.46 \pm 0.03)$ & $(17.3 \pm 1.3)$ & $(0.02 \pm 0.00)$ & $(0.05 \pm 0.01)$ & $(3.7 \pm 0.9)$ & $(1.6 \pm 0.3)$ \\
\hline & \multirow{4}{*}{ 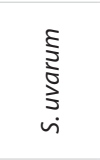 } & CBS395 & $(0.42 \pm 0.01)^{b}$ & $(18.9 \pm 0.4)^{a}$ & $(0.04 \pm 0.00)^{c *}$ & $(0.10 \pm 0.00)^{c *}$ & $(3.6 \pm 0.1)^{\mathrm{bc} *}$ & $(2.15 \pm 0.01)^{\mathrm{b} *}$ \\
\hline & & S103 & $(0.42 \pm 0.01)^{\mathrm{bc}}$ & $(18.8 \pm 0.7)^{\mathrm{a}}$ & $(0.05 \pm 0.00)^{c *}$ & $(0.11 \pm 0.01)^{\mathrm{c} *}$ & $(3.2 \pm 0.2)^{b}$ & $(1.8 \pm 0.3)^{\mathrm{ab}}$ \\
\hline & & SB42 & $(0.45 \pm 0.02)^{\mathrm{bc}}$ & $(17.8 \pm 0.8)^{\mathrm{a}}$ & $(0.03 \pm 0.00)^{\mathrm{b} *}$ & $(0.07 \pm 0.00)^{\mathrm{b} *}$ & $(1.61 \pm 0.05)^{\mathrm{a}}$ & $(1.5 \pm 0.2)^{\mathrm{ab}}$ \\
\hline & & Mean & $(0.43 \pm 0.02)$ & $(18.5 \pm 0.8)$ & $(0.04 \pm 0.01)$ & $(0.09 \pm 0.02)$ & $(2.8 \pm 0.9)$ & $(1.8 \pm 0.3)$ \\
\hline & \multirow{4}{*}{ 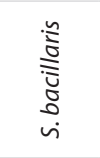 } & Y1667 & $(0.34 \pm 0.01)^{\mathrm{a} *}$ & $(23.1 \pm 0.4)^{\mathrm{b} *}$ & $(0.08 \pm 0.00)^{\mathrm{d} *}$ & $(0.23 \pm 0.02)^{\mathrm{d} *}$ & $(7.8 \pm 1.1)^{\mathrm{e}}$ & $(3.50 \pm 0.06)^{c *}$ \\
\hline & & Y1756 & $(0.35 \pm 0.01)^{\mathrm{a}}$ & $(22.8 \pm 0.9)^{\mathrm{b}}$ & $(0.07 \pm 0.00)^{\mathrm{d} *}$ & $(0.21 \pm 0.01)^{\mathrm{d} *}$ & $(6.7 \pm 0.6)^{\mathrm{de}}$ & $(3.7 \pm 0.2)^{c *}$ \\
\hline & & MLO & $(0.34 \pm 0.02)^{\mathrm{a} *}$ & $(22.9 \pm 1.1)^{\mathrm{b} *}$ & $(0.07 \pm 0.00)^{\mathrm{d} *}$ & $(0.20 \pm 0.00)^{\mathrm{d} *}$ & $(6.3 \pm 0.9)^{d}$ & $(3.8 \pm 0.2)^{\mathrm{c*}}$ \\
\hline & & Mean & $(0.34 \pm 0.01)$ & $(23.0 \pm 0.8)$ & $(0.07 \pm 0.00)$ & $(0.21 \pm 0.01)$ & $(6.9 \pm 1.0)$ & $(3.7 \pm 0.2)$ \\
\hline & \multicolumn{2}{|c|}{$\mathrm{LSD}_{5} \%$ S.c-other } & 0.02 & 0.9 & 0.01 & 0.01 & 0.76 & 0.21 \\
\hline & \multicolumn{2}{|c|}{$\mathrm{LSD}_{5}$ \%s.u.-s.b. } & 0.02 & 1.1 & 0.01 & 0.01 & 0.88 & 0.24 \\
\hline \multirow{17}{*}{320} & \multirow{7}{*}{ 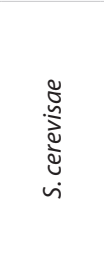 } & UVAFERMPM & $(0.45 \pm 0.01)^{\mathrm{A}}$ & $(17.5 \pm 0.5)^{\mathrm{AB}}$ & $(0.05 \pm 0.00)^{A *}$ & $(0.10 \pm 0.01)^{\mathrm{AB} *}$ & $(6.3 \pm 0.3)^{\mathrm{BCD}}$ & $(1.8 \pm 0.1)^{\mathrm{CD} *}$ \\
\hline & & SC57 & $(0.49 \pm 0.01)^{\mathrm{A}}$ & $(16.3 \pm 0.4)^{A}$ & $(0.04 \pm 0.00)^{A *}$ & $(0.08 \pm 0.01)^{A *}$ & $(5.6 \pm 0.4)^{\mathrm{BC}}$ & $(1.28 \pm 0.03)^{A B C}$ \\
\hline & & S701 & $(0.46 \pm 0.01)^{\mathrm{A}}$ & $(17.1 \pm 0.2)^{\mathrm{AB}}$ & $(0.04 \pm 0.00)^{\mathrm{A} *}$ & $(0.09 \pm 0.00)^{A *}$ & $(7.1 \pm 0.3)^{\mathrm{CD} *}$ & $(1.29 \pm 0.08)^{A B}$ \\
\hline & & RA100 & $(0.47 \pm 0.01)^{A}$ & $(16.7 \pm 0.5)^{A}$ & $(0.04 \pm 0.00)^{A *}$ & $(0.08 \pm 0.01)^{A *}$ & $(6.0 \pm 0.8)^{\mathrm{BCD}}$ & $(1.00 \pm 0.07)^{\mathrm{A}}$ \\
\hline & & UVAFERM228 & $(0.5 \pm 0.1)^{A}$ & $(17.3 \pm 3.6)^{\mathrm{AB}}$ & $(0.04 \pm 0.00)^{A *}$ & $(0.09 \pm 0.02)^{\mathrm{AB} *}$ & $(6.7 \pm 0.9)^{\mathrm{BCD} *}$ & $(1.4 \pm 0.2)^{\mathrm{AB}}$ \\
\hline & & SB12 & $(0.46 \pm 0.00)^{A B}$ & $(17.1 \pm 0.1)^{\mathrm{AB}}$ & $(0.05 \pm 0.00)^{A B *}$ & $(0.10 \pm 0.00)^{A B *}$ & $(8.0 \pm 0.1)^{\mathrm{DE*}}$ & $(1.20 \pm 0.06)^{A B *}$ \\
\hline & & Mean & $(0.47 \pm 0.04)$ & $(17.0 \pm 1.3)$ & $(0.04 \pm 0.00)$ & $(0.09 \pm 0.01)$ & $(6.6 \pm 0.9)$ & $(1.3 \pm 0.3)$ \\
\hline & \multirow{4}{*}{$\begin{array}{l}\frac{\xi}{3} \\
\frac{5}{5} \\
\frac{3}{J} \\
\dot{v i}\end{array}$} & CBS395 & $(0.38 \pm 0.05)^{C}$ & $(21.3 \pm 2.7)^{B}$ & $(0.06 \pm 0.00)^{\mathrm{C} *}$ & $(0.17 \pm 0.03)^{B *}$ & $(6.3 \pm 0.4)^{\mathrm{BCD} *}$ & $(1.6 \pm 0.1)^{\mathrm{BC} *}$ \\
\hline & & S103 & $(0.40 \pm 0.03)^{\mathrm{BC}}$ & $(19.8 \pm 1.3)^{A B}$ & $(0.05 \pm 0.00)^{\mathrm{BC} *}$ & $(0.14 \pm 0.00)^{B *}$ & $(4.7 \pm 0.5)^{A B}$ & $(1.34 \pm 0.09)^{A B C}$ \\
\hline & & SB42 & $(0.45 \pm 0.03)^{A B C}$ & $(17.4 \pm 1.2)^{\mathrm{AB}}$ & $(0.05 \pm 0.00)^{A B C *}$ & $(0.11 \pm 0.01)^{\mathrm{AB} *}$ & $(3.8 \pm 0.1)^{\mathrm{A}}$ & $(1.5 \pm 0.2)^{A B C}$ \\
\hline & & Mean & $(0.41 \pm 0.05)$ & $(19.5 \pm 2.3)$ & $(0.06 \pm 0.01)$ & $(0.14 \pm 0.03)$ & $(4.9 \pm 1.2)$ & $(1.5 \pm 0.2)$ \\
\hline & \multirow{4}{*}{ 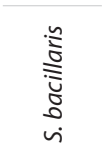 } & Y1667 & $(0.27 \pm 0.03)^{\mathrm{D} *}$ & $(29.2 \pm 2.6)^{C *}$ & $(0.10 \pm 0.00)^{\mathrm{D} *}$ & $(0.38 \pm 0.01)^{C_{*}}$ & $(9.4 \pm 1.4)^{\mathrm{E}}$ & $(2.6 \pm 0.2)^{\mathrm{D} *}$ \\
\hline & & Y1756 & $(0.30 \pm 0.03)^{\mathrm{D}}$ & $(25.3 \pm 0.5)^{c}$ & $(0.10 \pm 0.00)^{\mathrm{D} *}$ & $(0.33 \pm 0.03)^{C^{* *}}$ & $(9.9 \pm 1.5)^{\mathrm{E}}$ & $(2.8 \pm 0.3)^{\mathrm{D} *}$ \\
\hline & & MLO & $(0.30 \pm 0.00)^{\mathrm{D} *}$ & $(26.2 \pm 0.4)^{\mathrm{C} *}$ & $(0.10 \pm 0.00)^{\mathrm{D} *}$ & $(0.33 \pm 0.02)^{\mathrm{C*}}$ & $(7.6 \pm 0.8)^{\mathrm{DE}}$ & $(2.8 \pm 0.2)^{\mathrm{D} *}$ \\
\hline & & Mean & $(0.29 \pm 0.03)$ & $(26.9 \pm 2.2)$ & $(0.10 \pm 0.01)$ & $(0.34 \pm 0.03)$ & $(9.0 \pm 1.5)$ & $(2.7 \pm 0.2)$ \\
\hline & \multicolumn{2}{|c|}{$\mathrm{LSD}_{5 \% \mathrm{~S} . c \text {-other }}$} & 0.03 & 1.5 & 0.01 & 0.02 & 0.95 & 0.19 \\
\hline & \multicolumn{2}{|c|}{ LSD $_{5 \% \text { s.u.-s.b. }}$} & 0.04 & 1.8 & 0.01 & 0.02 & 1.09 & 0.22 \\
\hline
\end{tabular}

Values are mean of triplicate fermentations for each strain, bold values are species mean values of strain performance. After the Games-Howell post hoc comparison, the mean values in the same column are statistically different at $\mathrm{p}<0.05$; different lower-case letters indicate difference at $\gamma$ (sugar) $=220 \mathrm{~g} / \mathrm{L}$, capital letters at $\gamma($ sugar $)=320 \mathrm{~g} / \mathrm{L}$, and asterisks denotes the change induced by initial sugar concentration in the grape. $\mathrm{LSD}_{5 \%}$ values are the least significant differences between the mean values of species: LSD $_{5 \% \text {.c-other }}$ between Saccharomyces cerevisiae and the other two species, and $\mathrm{LSD}_{5 \% \text {. } . \text {.-s.b. }}$. between S. uvarum and Starmerella bacillaris. n.s. $=$ not significant

accordance with an earlier study (18). The L-succinic acid production was generally low in oenological terms (usual range $0.5-2.0 \mathrm{~g} / \mathrm{L}(27))$, but the yields were higher in the case of S. bacillaris than that of the other strains at both sugar concentrations (Table 2). Production of L-succinic acid by S. bacillaris Y1667 and MLO, S. cerevisiae UVAFERMPM and S. uvarum CBS395 seems to be influenced negatively by the initial sugar content. In certain vintages, the capability of a positive net organic acid production of a yeast is highly appreciated.

\section{Nitrogen utilisation}

The nitrogen demand of a certain species is highly important in winemaking, and it is even more highlighted in the case of non-Saccharomyces species in mixed fermentation, in terms of competition, balance or synergism $(4,28,29)$. Moreover, in grape juices with high sugar concentration, the appropriate amount of YAN could be crucial to complete the fermentation (21).

The composition of the YAN mimicked the grape juice composition. YAN concentration was adjusted to $300 \mathrm{mg} / \mathrm{L}$, well above the necessary level $(150-200 \mathrm{mg} / \mathrm{L}(21))$, but still below the optimal concentrations ( $400-500 \mathrm{mg} / \mathrm{L})$.

The YAN utilization by the strains was varying widely, between 30 and $63 \%$. At the higher sugar concentration, there was a significant decrease in YAN consumption by $S$. cerevisiae SC57 and RA100, S. uvarum CBS395 and S103 and all three $S$. bacillaris strains. Although the YAN consumption is reported to increase at higher sugar concentrations, in our investigations we used extremely high sugar concentration, 
Table 3. Some nitrogen-related properties of the different yeast species in chemically defined grape juice at initial $\gamma($ sugar $)=220$ and $320 \mathrm{~g} / \mathrm{L}$

\begin{tabular}{|c|c|c|c|c|c|c|}
\hline$\gamma($ sugar $) /(g / L)$ & Species & Strain & $\begin{array}{c}\gamma(\text { consumed YAN }) / \\
(\mathrm{mg} / \mathrm{L})\end{array}$ & $\begin{array}{c}\gamma(\text { consumed proline }) / \\
(\mathrm{mg} / \mathrm{L})\end{array}$ & $\begin{array}{c}\text { Specific YAN } \\
\text { consumption/sugar }\end{array}$ & $\begin{array}{c}\text { Specific YAN } \\
\text { consumption/biomass }\end{array}$ \\
\hline \multirow{17}{*}{220} & \multirow{7}{*}{ 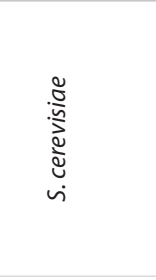 } & UVAFERMPM & $(141.2 \pm 13.0)^{\mathrm{ab}}$ & N.D. & $(0.48 \pm 0.06)^{\mathrm{a}}$ & $(0.52 \pm 0.05)^{a *}$ \\
\hline & & SC57 & $(196.3 \pm 4.6)^{\mathrm{ab} *}$ & $(0.2 \pm 0.3)^{\mathrm{a}}$ & $(0.73 \pm 0.02)^{\mathrm{bc} *}$ & $(0.72 \pm 0.02)^{\mathrm{bc}}$ \\
\hline & & S701 & $(122.9 \pm 5.2)^{c}$ & N.D. & $(0.40 \pm 0.02)^{\mathrm{a}}$ & $(0.61 \pm 0.03)^{\mathrm{ab} *}$ \\
\hline & & RA100 & $(191.0 \pm 2.5)^{\mathrm{b} *}$ & N.D. & $(0.79 \pm 0.03)^{\mathrm{cd} *}$ & $(1.60 \pm 0.02)^{\mathrm{e}}$ \\
\hline & & UVAFERM228 & $(143.4 \pm 13.5)^{\mathrm{ab}}$ & N.D. & $(0.51 \pm 0.07)^{\mathrm{ab}}$ & $(0.7 \pm 0.6)^{\mathrm{ab}}$ \\
\hline & & SB12 & $(129.6 \pm 4.7)^{c}$ & N.D. & $(0.42 \pm 0.02)^{\mathrm{a}}$ & $(0.95 \pm 0.03)^{d}$ \\
\hline & & Mean & $(154.1 \pm 31.1)$ & $(0.03 \pm 0.1)$ & $(0.6 \pm 0.2)$ & $(0.8 \pm 0.4)$ \\
\hline & \multirow{4}{*}{\begin{tabular}{l}
$\frac{5}{3}$ \\
$\frac{5}{0}$ \\
\multirow{3}{*}{} \\
vi
\end{tabular}} & CBS395 & $(211.7 \pm 4.4)^{\mathrm{d} *}$ & N.D. & $(1.23 \pm 0.01)^{\mathrm{e} *}$ & $(1.76 \pm 0.04)^{\mathrm{e}}$ \\
\hline & & $\mathrm{S} 103$ & $(219.6 \pm 2.3)^{\mathrm{d} *}$ & N.D. & $(1.28 \pm 0.08)^{e *}$ & $(1.26 \pm 0.04)^{e *}$ \\
\hline & & SB42 & $(139.4 \pm 14.2)^{\mathrm{ab}}$ & N.D. & $(0.59 \pm 0.08)^{\mathrm{ab}}$ & $(0.84 \pm 0.01)^{\mathrm{cd} *}$ \\
\hline & & Mean & $(190.3 \pm 39.0)$ & N.D. & $(1.0 \pm 0.4)$ & $(1.3 \pm 0.2)$ \\
\hline & \multirow{4}{*}{ 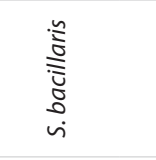 } & Y1667 & $(138.5 \pm 15.7)^{\mathrm{a} *}$ & N.D. & $(1.0 \pm 0.1)^{\mathrm{de} *}$ & $(0.67 \pm 0.08)^{b *}$ \\
\hline & & Y1756 & $(172.9 \pm 2.6)^{\mathrm{ab} *}$ & $(3.4 \pm 3.9)^{\mathrm{a*}}$ & $(1.24 \pm 0.02)^{2 *}$ & $(0.86 \pm 0.08)^{d *}$ \\
\hline & & MLO & $(173.1 \pm 7.0)^{\mathrm{ab} *}$ & $(2.5 \pm 4.4)^{\mathrm{a} *}$ & $(1.3 \pm 0.01)^{\mathrm{e} *}$ & $(0.9 \pm 0.4)^{d *}$ \\
\hline & & Mean & $(161.5 \pm 19.3)$ & $(2.0 \pm 3.3)$ & $(1.2 \pm 0.2)$ & $(0.8 \pm 0.1)$ \\
\hline & LSD $_{5 \% 5 . c-\text { other }}$ & & n.s & n.s & 0.18 & n.s. \\
\hline & $\operatorname{LSD}_{5 \% \text { s.u.-s.b. }}$ & & n.s. & n.s. & 0.21 & n.s. \\
\hline \multirow{17}{*}{320} & \multirow{7}{*}{ 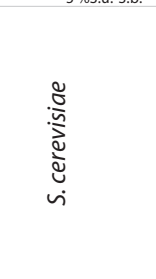 } & UVAFERMPM & $(144.7 \pm 6.5)^{A}$ & $(12.3 \pm 6.0)^{\mathrm{AB} *}$ & $(0.58 \pm 0.08)^{A B}$ & $(1.21 \pm 0.06)^{A *}$ \\
\hline & & SC57 & $(142.1 \pm 4.7)^{\mathrm{A} *}$ & N.D. & $(0.48 \pm 0.01)^{\mathrm{ABC} *}$ & $(0.81 \pm 0.03)^{\mathrm{B}}$ \\
\hline & & S701 & $(125.2 \pm 6.2)^{\mathrm{B}}$ & $(4.0 \pm 2.5)^{\mathrm{AB}}$ & $(0.40 \pm 0.04)^{\mathrm{A}}$ & $(0.74 \pm 0.04)^{C_{*}}$ \\
\hline & & RA100 & $(142.6 \pm 3.8)^{\mathrm{CD} *}$ & $(6.7 \pm 6.8)^{\mathrm{AB}}$ & $(0.50 \pm 0.03)^{A B C *}$ & $(1.49 \pm 0.04)^{\mathrm{D}}$ \\
\hline & & UVAFERM 228 & $(136.4 \pm 6.9)^{\mathrm{CD}}$ & $(13.8 \pm 0.7)^{\mathrm{B} *}$ & $(0.54 \pm 0.02)^{\mathrm{ABC}}$ & $(0.58 \pm 0.03)^{\mathrm{E}}$ \\
\hline & & SB12 & $(119.8 \pm 8.2)^{\mathrm{AB}}$ & $(0.1 \pm 0.2)^{A}$ & $(0.39 \pm 0.04)^{A}$ & $(0.89 \pm 0.06)^{\mathrm{B}}$ \\
\hline & & Mean & $(135.1 \pm 11.0)$ & $(6.1 \pm 6.8)$ & $(0.48 \pm 0.08)$ & $(1.0 \pm 0.3)$ \\
\hline & \multirow{4}{*}{ 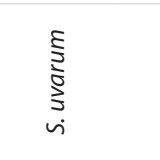 } & CBS395 & $(119.0 \pm 9.6)^{\mathrm{C*}}$ & $(9.9 \pm 9.7)^{\mathrm{AB} *}$ & $(0.64 \pm 0.03)^{\complement *}$ & $(1.3 \pm 0.1)^{\mathrm{A}}$ \\
\hline & & $\mathrm{S} 103$ & $(114.5 \pm 8.5)^{\mathrm{C} *}$ & $(0.1 \pm 0.3)^{\mathrm{A}}$ & $(0.52 \pm 0.05)^{\mathrm{ABC} *}$ & $(0.91 \pm 0.07)^{B *}$ \\
\hline & & SB42 & $(126.6 \pm 1.3)^{\mathrm{CD}}$ & N.D. & $(0.53 \pm 0.01)^{\mathrm{ABC}}$ & $(0.65 \pm 0.01)^{\mathrm{CE} *}$ \\
\hline & & Mean & $(120.1 \pm 8.3)$ & $(3.3 \pm 6.9)$ & $(0.56 \pm 0.06)$ & $(0.9 \pm 0.3)$ \\
\hline & \multirow{4}{*}{ 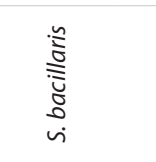 } & Y1667 & $(95.7 \pm 11.0)^{\mathrm{D} *}$ & $(18.6 \pm 7.6)^{\mathrm{B} *}$ & $(0.6 \pm 0.1)^{\mathrm{BC} *}$ & $(0.42 \pm 0.05)^{\mathrm{F} *}$ \\
\hline & & Y1756 & $(88.9 \pm 3.4)^{\mathrm{D} *}$ & $(17.6 \pm 4.4)^{\mathrm{B} *}$ & $(0.46 \pm 0.05)^{\mathrm{AB} *}$ & $(0.42 \pm 0.02)^{\mathrm{F} *}$ \\
\hline & & MLO & $(85.0 \pm 12.9)^{\mathrm{E*}}$ & $(18.3 \pm 5.6)^{\mathrm{B} *}$ & $(0.5 \pm 0.1)^{\mathrm{ABC} *}$ & $(0.44 \pm 0.07)^{F *}$ \\
\hline & & Mean & $(89.9 \pm 9.8)$ & $(18.2 \pm 8.4)$ & $(0.50 \pm 0.09)$ & $(0.43 \pm 0.04)$ \\
\hline & \multicolumn{2}{|l|}{$\mathrm{LSD}_{5 \% \text { s.c-other }}$} & 8.4 & 6.0 & n.s. & n.s. \\
\hline & \multicolumn{2}{|l|}{$\mathrm{LSD}_{5 \% \text { s.u.-s.b. }}$} & 9.7 & 7.0 & n.s. & n.s. \\
\hline
\end{tabular}

Values are mean of triplicate fermentations for each strain, bold values are species mean values of strain performance. After the Games-Howell post hoc comparison, the mean values in the same column are statistically different at $\mathrm{p}<0.05$; different lower-case letters indicate difference at $\gamma$ (sugar) $=220 \mathrm{~g} / \mathrm{L}$, capital letters at $\gamma$ (sugar) $=320 \mathrm{~g} / \mathrm{L}$, and asterisks denotes the change induced by initial sugar concentration in the grape. $\mathrm{LSD}_{5 \%}$ values are the least significant differences between the mean values of species: $\mathrm{LSD}_{5 \%}$ s.c-other between Saccharomyces cerevisiae and the other two species, and $\mathrm{LSD}_{5} \%$ s.u.s.b. between . uvarum and Starmerella bacillaris. n.s. $=$ not significant. YAN=yeast assimilable nitrogen

where the nitrogen metabolism might be repressed to some extent (Table 3). The specific YAN consumption data show (Table 3 ) that the sugar-induced change in its consumption by S. bacillaris strains $(0.43-0.81 \mathrm{mg} / \mathrm{g})$ was similar to $S$. uvarum CBS395 $(0.59 \mathrm{mg} / \mathrm{g})$ and S103 $(0.76 \mathrm{mg} / \mathrm{g})$. The absolute YAN consumption by $S$. bacillaris was comparable with that of Saccharomyces species at lower sugar concentration, which corresponds to an earlier experiment (30). At high sugar concentration, S. bacillaris strains showed a moderate demand for nitrogen. This behaviour could be useful in mixed fermentations in juices where the initial sugar concentration is high or even extremely high.

Proline uptake was practically undetected at $220 \mathrm{~g} / \mathrm{L}$ sugar, but it was taken up poorly at the higher sugar concentration (Table 3). This trend was prevalent for all the species, but moderate and strongly strain-dependent in the Saccharomyces species, involving S. cerevisiae UVAFERMPM, UVAFERM 228 and S. uvarum CBS395. However, there was a more considerable increase in the proline utilization at the higher sugar level in the case of all strains of Starmerella bacillaris. Proline, which would be an abundant source of nitrogen in every grape juice, is not or is negligibly utilized by wine yeasts due to an inhibition under anaerobic conditions $(29,30)$. Our results are consistent with this view at the normal sugar concentration, but an improved proline utilization might function at extreme sugar level, particularly in S. bacillaris.

\section{Multivariate performance assessment}

The sugar-induced changes in the above-described metabolites and nitrogen utilization of the investigated yeasts 
should be taken into consideration in one model. Supplementary Fig. S1 shows the results of discriminant function analysis, with three significant functions. The strains were clearly distinct at different sugar concentrations.

Function 1, ethanol, did not group with either factor. Function 2 represented the factors that were sugar-dependent in a positive manner, namely volatile acidity and proline utilization, while Function 3 comprised factors negatively influenced by the sugar level, namely consumption of YAN and L-malic acid, and production of L-succinic acid. The performance of S. uvarum strains was overall considerably closer to that of S. cerevisiae than of S. bacillaris strains. The S. cerevisiae strains formed the most scattered group, which indicates their high intraspecies diversity, in terms of their altered metabolite production due to extremely high sugar concentration.

\section{CONCLUSIONS}

The high sugar-driven changes in the major fermentation metabolite pattern and nitrogen utilization pointed out an altered behaviour of the investigated species. The overall performance of Saccharomyces uvarum strains was close to S. cerevisiae in general; however, with clear differences in certain properties, e.g. volatile acid and glycerol production, while Starmerella bacillaris strains exhibited distinct, sometimes inverse behaviour than the Saccharomyces species. The effects of the extremely high sugar concentration on the oenologically beneficial traits were the most pronounced in $S$. bacillaris.

Regarding the nitrogen-related features of the S. bacillaris strains, namely the moderate yeast assimilable nitrogen demand and the noticeable ability to utilize proline at higher sugar level, this species could be a promising match in a mixed fermentation with $S$. cerevisiae, especially for grape juices with high or even extremely high sugar concentration.

In this experiment, the adequacy of this non-Saccharomyces species for winemaking, in combination with a suitable Saccharomyces yeast, is highlighted. Moreover, the results suggest that $S$. bacillaris possesses a more active adaptation mechanism to extreme sugar concentration. Undoubtedly, a more detailed investigation would be useful in the future focusing on the minor and volatile metabolites with an even wider strain set.

\section{ACKNOWLEDGEMENTS}

The authors wish to thank the valuable help of Andrea Pomázi and Vivien Kormos during the experimental phase and the Dyagnosticum Kft. for the NMR measurements.

\section{CONFLICT OF INTEREST}

The authors declare no conflict of interest.

\section{SUPPLEMENTARY MATERIAL}

All supplementary material is available at www.ftb.com.hr.

\section{ORCID ID}

B. Oláhné Horváth (1) https://orcid.org/0000-0001-8809-546X

D. Nyitrainé Sárdy @ https://orcid.org/0000-0002-6132-1671

N. Kellner (1) https://orcid.org/0000-0002-3798-0116

I. Magyar (ㄱ https://orcid.org/0000-0002-0228-1710

\section{REFERENCES}

1. Rollero S, Bloem A, Ortiz-Julien A, Camarasa C, Divol B. Altered fermentation performances, growth, and metabolic footprints reveal competition for nutrients between yeast species inoculated in synthetic grape juice-like medium. Front Microbiol. 2018;9:196.

https://doi.org/10.3389/fmicb.2018.00196

2. Englezos V, Rantsiou K, Cravero F, Torchio F, Ortiz-Julien A, Gerbi V, et al. Starmerella bacillaris and Saccharomyces cerevisiae mixed fermentations to reduce ethanol content in wine. Appl Microbiol Biotechnol. 2016;100(12):5515-26. https://doi.org/10.1007/s00253-016-7413-z

3. Rossouw D, Bauer FF. Exploring the phenotypic space of non-Saccharomyces wine yeast biodiversity. Food Microbiol. 2016;55:32-46.

https://doi.org/10.1016/j.fm.2015.11.017

4. Ciani M, Capece A, Comitini F, Canonico L, Siesto G, Romano P. Yeast interactions in inoculated wine fermentation. Front Microbiol. 2016;7:555.

https://doi.org/10.3389/fmicb.2016.00555

5. Suárez-Lepe JA, Morata A. New trends in yeast selection for winemaking. Trends Food Sci Technol. 2012;23(1):39-50. https://doi.org/10.1016/j.tifs.2011.08.005

6. Sipiczki M. Candida zemplinina sp nov., an osmotolerant and psychrotolerant yeast that ferments sweet botrytized wines. Int J Syst Evol Microbiol. 2003;53(Pt 6):2079-83. https://doi.org/10.1099/ijs.0.02649-0

7. Duarte FL, Pimentel NH, Teixeira A, Fonseca Á. Saccharomyces bacillaris is not a synonym of Candida stellata: Reinstatement as Starmerella bacillaris comb. nov. Antonie Van Leeuwenhoek. 2012;102(4):653-8. https://doi.org/10.1007/s10482-012-9762-7

8. Mills DA, Johannsen EA, Cocolin L. Yeast diversity and persistence in botrytis-affected wine fermentations. Appl Environ Microbiol. 2002;68(10):4884-93. https://doi.org/10.1128/AEM.68.10.4884-4893.2002

9. Magyar I, Tóth T. Comparative evaluation of some oenological properties in wine strains of Candida stellata, Candida zemplinina, Saccharomyces uvarum and Saccharomyces cerevisiae. Food Microbiol. 2011;28(1):94-100.

https://doi.org/10.1016/j.fm.2010.08.011

10. Masneuf-Pomarede I, Juquin E, Miot-Sertier C, Renault P, Laizet Y, Salin F, et al. The yeast Starmerella bacillaris (synonym Candida zemplinina) shows high genetic diversity in winemaking environments. FEMS Yeast Res. 
2015;15(5):fov045.

https://doi.org/10.1093/femsyr/fov045

11. Tofalo R, Schirone M, Torriani S, Rantsiou K, Cocolin L, Perpetuini G, Suzzi G. Diversity of Candida zemplinina strains from grapes and Italian wines. Food Microbiol. 2012;29(1):18-26.

https://doi.org/10.1016/j.fm.2011.08.014

12. Englezos V, Giacosa S, Rantsiou K, Rolle L, Cocolin L. Starmerella bacillaris in winemaking: Opportunities and risks. Curr Opin Food Sci. 2017;17:30-5.

https://doi.org/10.1016/j.cofs.2017.08.007

13. Kemsawasd V, Viana T, Ardö Y, Arneborg N. Influence of nitrogen sources on growth and fermentation performance of different wine yeast species during alcoholic fermentation. Appl Microbiol Biotechnol. 2015;99(23):10191-207. https://doi.org/10.1007/s00253-015-6835-3

14. Vilanova M, Ugliano M, Varela C, Siebert T, Pretorius IS, Henschke PA. Assimilable nitrogen utilisation and production of volatile and non-volatile compounds in chemically defined medium by Saccharomyces cerevisiae wine yeasts. Appl Microbiol Biotechnol. 2007;77(1):145-57. https://doi.org/10.1007/s00253-007-1145-z

15. Nisiotou A, Sgouros G, Mallouchos A, Nisiotis CS, Michaelidis C, Tassou C, Banilas G. The use of indigenous Saccharomyces cerevisiae and Starmerella bacillaris strains as a tool to create chemical complexity in local wines. Food Res Int. 2018;111:498-508.

https://doi.org/10.1016/j.foodres.2018.05.035

16. Englezos V, Rantsiou K, Cravero F, Torchio F, Pollon M, Fracassetti $D$, et al. Volatile profile of white wines fermented with sequential inoculation of Starmerella bacillaris and Saccharomyces cerevisiae. Food Chem. 2018;257:350-60. https://doi.org/10.1016/j.foodchem.2018.03.018

17. Lencioni L, Taccari M, Ciani M, Domizio P. Zygotorulaspora florentina and Starmerella bacillaris in multistarter fermentation with Saccharomyces cerevisiae to reduce volatile acidity of high sugar musts. Aust J Grape Wine Res. 2018;24(3):368-72.

https://doi.org/10.1111/ajgw.12327

18. Rantsiou K, Englezos V, Torchio F, Risse PA, Cravero F, Gerbi $V$, et al. Modeling of the fermentation behavior of Starmerella bacillaris. Am J Enol Viticult. 2017;68(3):378-85.

https://doi.org/10.5344/ajev.2017.16108

19. Rantsiou K, Dolci P, Giacosa S, Torchio F, Tofalo R, Torriani S, et al. Candida zemplinina can reduce acetic acid produced by Saccharomyces cerevisiae in sweet wine fermentations. Appl Environ Microbiol. 2012;78(6):1987-94. https://doi.org/10.1128/AEM.06768-11

20. Zott K, Claisse O, Lucas P, Coulon J, Lonvaud-Funel A, Masneuf-Pomarede I. Characterization of the yeast ecosystem in grape must and wine using real-time PCR. Food
Microbiol. 2010;27(5):559-67.

https://doi.org/10.1016/j.fm.2010.01.006

21. Henschke PA, Jiranek V. Yeasts - Metabolism of nitrogen compounds. In: Fleet GH, editor. Wine microbiology and biotechnology. London, UK: Taylor \& Francis, Ltd; 1993. pp. 77-164.

22. Method OIV-MA-AS312-01A. Alcoholic strength by volume. Compendium of International Methods of Analysis of Wines and Musts ( 2 vol.). Paris, France: The International Organisation of Vine and Wine (OIV); 2019. Available from: http://www.oiv.int/public/medias/5158/oiv-ma-as312-01a. pdf.

23. Method OIV-MA-AS313-01. Total acidity. Compendium of International Methods of Analysis of Wines and Musts (2 vol.). Paris, France: The International Organisation of Vine and Wine (OIV); 2019.-Available from: http://www.oiv.int/ public/medias/3732/oiv-ma-as313-02.pdf.

24. Method OIV-MA-AS311-01A. Reducing substances. Compendium of International Methods of Analysis of Wines and Musts (2 vol.). Paris, France: The International Organisation of Vine and Wine (OIV); 2019.-Available from: http://www. oiv.int/public/medias/2481/oiv-ma-as311-01a.pdf.

25. Godelmann R, Fang F, Humpfer E, Schütz B, Bansbach M, Schafer $\mathrm{H}$, Spraul M. Targeted and nontargeted wine analysis by ${ }^{1} \mathrm{H}$ NMR spectroscopy combined with multivariate statistical analysis. Differentiation of important parameters: Grape variety, geographical origin, year of vintage. J Agric Food Chem. 2013;61(23):5610-9.

https://doi.org/10.1021/jf400800d

26. IBM SPSS Statistics for Windows, v. 23.0, IBM Corp., Armonk, NY, USA; 2016. Available from: https://www.ibm.com/analytics/spss-statistics-software.

27. Radler F. Yeasts - Metabolism of organic acids. In: Fleet GH, editor. Wine microbiology and biotechnology. London, UK: Taylor \& Francis, Ltd; 1993. pp. 165-82.

28. Gobert A, Tourdot-Maréchal R, Morge C, Sparrow C, Liu Y, Quintanilla-Casas B, et al. Non-Saccharomyces yeasts nitrogen source preferences: Impact on sequential fermentation and wine volatile compounds profile. Front Microbiol. 2017;8:2175.

https://doi.org/10.3389/fmicb.2017.02175

29. Prior KJ, Bauer FF, Divol B. The utilisation of nitrogenous compounds by commercial non-Saccharomyces yeasts associated with wine. Food Microbiol. 2019;79:75-84.

https://doi.org/10.1016/j.fm.2018.12.002

30. Englezos V, Cocolin L, Rantsiou K, Ortiz-Julien A, Bloem A, Dequin S, Camarasa C. Specific phenotypic traits of Starmerella bacillaris related to nitrogen source consumption and central carbon metabolite production during wine fermentation. Appl Environ Microbiol. 2018;84(16):e00797-18. https://doi.org/10.1128/AEM.00797-18 